\title{
EFECTO DEL TAMAÑO DE CONTENEDOR SOBRE EL CRECIMIENTO EN VIVERO Y COMPORTAMIENTO EN TERRENO DE PLANTAS DE Quillaja saponaria Mol. ESTABLECIDAS EN FLORIDA, REGIÓN DEL BIOBÍO
}

\author{
Quiroz, Iván ${ }^{5}$; González, Marta; Hernández, Andrés; \\ Soto, Hernán; García, Edison y Pincheira, Matías.
}

\section{RESUMEN}

El quillay (Quillaja saponaria Mol.), es una especie nativa y endémica de la zona central de Chile, se distribuye naturalmente entre los 30 $30^{\prime}$ y los $38^{\circ} \mathrm{LS}$, regiones de Coquimbo y La Araucanía, pertenece al Tipo Forestal Esclerófilo, y es una de las especies arbóreas de mayor importancia en este tipo forestal por su abundancia y amplia distribución.

Pese a no tener grandes restricciones para su desarrollo, existen actualmente problemas en el estado de conservación de la especie en formaciones naturales debido a las fuertes intervenciones a que ha sido sometida para la obtención de productos no maderables destinados a los mercados farmacéuticos y cosmetológicos.

Considerando su potencial como especie para programas de forestación, resulta de interés revisar su propagación en viveros, dado que la heterogeneidad de las plantas que los viveros producen cada año, principalmente en sus características morfológicas, hace recomendable conocer estándares de calidad de plantas que permitan asegurar un exitoso establecimiento plantaciones.

El presente trabajo entrega resultados obtenidos por el Centro Tecnológico de la Planta Forestal, un centro tecnológico del Instituto Forestal, en ensayos de producción de plantas de quillay, incluyendo la comparación y análisis de producciones realizadas en diversas investigaciones, con el objeto determinar parámetros morfológicos e indicadores de calidad de plantas de la especie, y evaluando el comportamiento de plantas de quillay establecidas en terreno y producidas en contenedores de diversos volúmenes.

Se consideró 10 tratamientos, dados por diversos tipos de bandejas con diferentes cantidades de contenedores ( 53 a $310 \mathrm{~cm}^{3}$ de capacidad). Se concluye que el volumen 0 capacidad del contenedor es una variable que tiene una buena correlación con las características morfológicas de las plantas producidas en vivero, siendo el peso seco y el DAC (diámetro del cuello) las variables con una mayor correlación y esta se da principalmente dentro de un rango bien definido de volumen. No obstante, la cantidad de contenedores por bandeja, que incorpora indirectamente la variable densidad o cantidad de plantas por unidad de superficie, incide negativamente.

Existe una alta correlación con las variables morfológicas de las plantas en etapa de vivero, es decir a medida que aumenta el volumen y profundidad del contenedor, mayores son los valores de dichas variables, característica que se presenta con mayor notoriedad en la longitud de la raíz. Por el contrario, estas variables disminuyen a medida que aumenta el número de cavidades por almaciguera y por unidad de superficie, principalmente en la altura y el diámetro.

El efecto de los mejores tratamientos se observa también en las plantaciones en terreno.

Palabras clave: Quillay, Quillaja saponaria, Técnicas de vivero

5 Instituto Forestal, Sede Biobío. ivan.quiroz@infor.cl 


\section{SUMMARY}

Quillay (Quillaja saponaria Mol.) is a native and endemic species in Central Chile, its natural distribution is between $30^{\circ} 30^{\prime}$ and $30^{\circ} \mathrm{SL}$, Coquimbo to La Araucanía regions of the country. The species is part of the Bosque Esclerófilo type being one of the most important trees on this type of forests due to its abundance and wide distribution.

Despite de species has not major development problems, there are some conservation problems in native forests because of the high pressure over the resource to obtain non wood products oriented to the pharmaceutical and cosmetology industries.

Considering its importance as a suitable species for afforestation programs, a nursery techniques review is necessary. Currently a high heterogeneity can be observed on the seedlings production in nurseries each year, mainly regarding to morphological properties, and the produced seedlings could be not the best material towards an appropriate plantation establishment.

This paper shows some results on nursery production trials, obtained at the INFOR's Technological Forest Seedling Centre, using different container types to produce the seedlings and evaluating the results both, at the end of the nursery period and after the field establishment .

Ten treatments were tested considered different types of containers varying the volume and the number of then per area unit. It is concluded that the volume is a high correlated variable with the seedling morphological properties, being the dry weight and the neck diameter the most correlated characteristics. As bigger is the container in terms of volume and deep better are the results. On the contrary, as more containers are per area unit, variable values decrease.

The positive effect of the better treatments is also observed after planting on the field.

Key words: Quillay, Quillaja saponaria, Nursery techniques 


\section{INTRODUCCIÓN}

El quillay, es una especie endémica de la zona central de Chile y se distribuye naturalmente entre los $30^{\circ} 30^{\prime}$ y los $38^{\circ} \mathrm{LS}$, esto es entre las regiones de Coquimbo y La Araucanía (Enciclopedia de la Flora Chilena, 2012).

Esta especie pertenece al Tipo Forestal Esclerófilo, que se caracteriza por la presencia dominante de especies de hojas duras, de dimensiones tales que se pueden calificar de arbustivas o arbóreas. Es una de las especies arbóreas de mayor importancia en el bosque esclerófilo, por su abundancia y amplia distribución en el país (INFOR-INDAP-FIA 2000). No obstante, además es una especie frecuente en los Tipos Forestales, Palma Chilena, Roble-Hualo y Ciprés de la Cordillera (Rodríguez et al. 1983). Corresponde a un árbol o arbusto, que alcanza entre 20 y $30 \mathrm{~m}$ de altura y 1,5 m de DAP, en suelos profundos y planos. Su follaje es siempreverde y su corteza es cenicienta. Su tronco es cilíndrico y normalmente se ramifica entre los 2 y $5 \mathrm{~m}$ del suelo con gran frondosidad, por lo que es común emplearlo en el campo como protección de ganado.

Vita (1974, cit. por INFOR-INDAP-FIA 2000) señala que debido a su plasticidad la especie es capaz de desarrollarse tanto en condiciones de temperaturas moderadas, como soportando calores en verano e intensos fríos en invierno. El quillay es una especie adaptada para vivir en sitios pobres, secos y cálidos. Crece bien en suelos degradados, con pendiente y asoleados, aunque su mayor desarrollo lo alcanza en suelos profundos y planos.

En el extremo septentrional de su distribución (Ovalle, Región de Coquimbo), el clima se caracteriza por presentar 10 meses de sequía y $150 \mathrm{~mm}$ anuales de precipitación, y en el extremo meridional (Collipulli, Región de la Araucanía) presenta 3 meses de sequía y $1.500 \mathrm{~mm}$ anuales de precipitación. Quillay presenta algunas estrategias para enfrentar periodos de estrés. En este sentido, un estudio realizado por Donoso et al. (2011) en plantas de quillay sometidas a estrés hídrico concluye que en el periodo de mayor restricción hídrica, quillay no presentó un mecanismo claro de ajuste al déficit hídrico en términos fisiológicos, pero si en términos morfológicos, reduciendo su biomasa foliar en $55 \%$ e incrementando su biomasa de raíces para reducir la pérdida hídrica. Así, presentó una reducción significativa de la fotosíntesis neta, conductancia estomática y transpiración frente a riego restringido.

La especie se encuentra en altitudes desde los $100 \mathrm{msnm}$ en la Cordillera de la Costa hasta los $1.500 \mathrm{msnm}$ en la Cordillera de los Andes, pudiendo llegar hasta los $2.000 \mathrm{msnm}$ (INFOR-INDAP-FIA 2000).

La corteza es utilizada históricamente como detergente, debido a la gran cantidad de saponina que contiene. Es una especie con buen potencial para programas de reforestación en suelos áridos y es ampliamente utilizada como ornamental (INFOR-INDAP-FIA 2000).

Los árboles de quillay del bosque esclerófilo se explotan intensamente para la extracción de saponinas desde la corteza, fustes y ramas. Además, se obtienen otros productos como carbón y leña. Las saponinas son tensoactivos naturales que se utilizan para la elaboración de espumantes de bebidas, preparación de películas fotográficas, cosméticos y fármacos. La mayor concentración de saponinas se encuentra en la corteza y desde esta se han extraído tradicionalmente, pero en la actualidad existe la tecnología para extraer saponinas también desde fustes y ramas. Para satisfacer la demanda mundial de saponina, se estima que se cortan y descortezan 60.000 quillayes de 30-50 años, los que producen $1.000 \mathrm{t}$ de corteza. A una densidad promedio de 15 árboles/ha se intervienen aproximadamente 4.000 ha cada año de bosque esclerófilo. Sin embargo, utilizando toda la biomasa es posible reducir el número de árboles que se cosecha cada año. Investigaciones realizadas por la Pontificia Universidad Católica de Chile han encontrado diversos usos no tradicionales del quillay, algunos que ya se encuentran en fase comercial, como el QL AGRI®, un nematicída apto para agricultura tradicional y orgánica. También el $\mathrm{QP} \AA$, un aditivo que mejora la alimentación y el metabolismo animal, reduciendo los malos 
olores en las fecas. Otro ejemplo es el Mistop $\AA$, un tensoactivo usado para la reducción de neblina ácida en procesos de electro-obtención de cobre y zinc (http://www.fondef.cl/content/view/229/195).

Cabe señalar que pese a no tener grandes restricciones para su desarrollo, existen actualmente problemas en el estado de conservación de la especie en formaciones naturales debido a las fuertes intervenciones a que ha sido sometida para la obtención de productos no maderables destinados a los mercados farmacéuticos y cosmetológicos (Santelíces y Bobadilla, 1997).

Dado lo anterior y considerando su potencial como especie para programas de forestación, resulta de interés revisar su propagación en viveros, donde la heterogeneidad de las plantas que los viveros producen cada año, principalmente en las características morfológicas, hace recomendable conocer estándares de calidad de plantas que permitan asegurar un exitoso establecimiento plantaciones.

Los atributos morfológicos, según Rose et al. (1990), pueden correlacionarse exitosamente con la supervivencia y el crecimiento inicial de muchas especies forestales. La altura total de la planta y el diámetro de cuello, junto con la relación diámetro de cuello - altura (DAC/H), fueron los primeros atributos utilizados. No obstante, la morfología de la planta no es una característica por si sola que defina su calidad. El uso de métodos ecofisiológicos clásicos de medición de tasas de fotosíntesis, respiración y transpiración, conductividad estomática y potencial hídrico, al igual que contenido y relación de pigmentos fotosintéticos (clorofila y carotenoides) o la concentración de metabolitos que aparecen en condiciones de estrés, son variables que complementan la toma de decisiones en el caso de definir qué tipo de planta producir.

En cuanto a las variables morfológicas, el tamaño del contenedor tiene una correlación directa con las plantas que se producirán. A mayor volumen del contenedor las magnitudes de altura y diámetro de cuello también aumentan (Domínguez et al. 1997, 2000). Otra característica importante es la profundidad del contenedor. Contenedores de mayor profundidad y de sección estrecha pueden restringir la aireación del sustrato y las raíces, deteriorando la calidad de las plantas. No obstante, la mayor longitud o profundidad del contenedor puede ser una característica deseable en la producción de especies que desarrollan una fuerte raíz pivotante (Domínguez 1997). Incluso el color del contenedor, así como el material que los conforma, puede influir en alguna medida sobre la desecación de los cepellones, los colores oscuros provocan un aumento de temperatura y por lo tanto mayor evaporación, sobre todo en las plantas expuestas directamente al sol, afectando la supervivencia y desarrollo de éstas (Domínguez 2000). Por consiguiente, la selección del contenedor más apropiado, dependerá del balance entre la calidad de la planta producida y el costo de su producción (Arizaleta y Pire 2008).

El presente trabajo entrega resultados obtenidos por el Centro Tecnológico de la Planta Forestal, un centro tecnológico del Instituto Forestal, en ensayos de producción de plantas de quillay, incluyendo la comparación y análisis de producciones realizadas en diversas investigaciones, con el objeto determinar parámetros morfológicos e indicadores de calidad de plantas de la especie, y evaluando el comportamiento de plantas de quillay establecidas en terreno y producidas en contenedores de diversos volúmenes.

\section{MATERIAL Y MÉTODO}

El estudio de evaluación de efecto del tamaño de contendor sobre el crecimiento de Quillaja saponaria fue ejecutado en dos fases secuenciales, la primera de crecimiento en vivero en las instalaciones del Instituto Forestal, ubicado en la comuna de San Pedro de la Paz en Concepción, y la segunda de crecimiento en terreno en una plantación establecida en la comuna de Florida, región de Biobío. Se utilizaron semillas de quillay colectadas el año 2009 , procedentes de la zona de Rapilermo, comuna de Curepto, Región del Maule (3511' 59' LS; $71^{\circ} 46^{\prime}$ 59" LO, $324 \mathrm{msnm}$ ). Las semillas se remojaron en agua por 24 horas, como tratamiento germinativo previo. 


\section{Crecimiento en Vivero}

\section{- Producción de Plantas}

\section{Siembra}

La siembra de las semillas de quillay se llevó a cabo en el vivero de la sede Bio Bio de INFOR en Concepción ( $36^{\circ}$ 50' 61" LS; 73 07' 56" LO, 18 msnm), el 4 de junio de 2009 en diversos tipos de contenedores (Cuadro $\mathrm{N}^{\circ} 1$ ). El sustrato utilizado fue corteza de pino compostada de granulometría G-10. Las bandejas sembradas fueron tratadas con aspersiones semanales de una solución de fungicida en agua compuesta por una mezcla en igual proporción de Benlate y Captan a razón de $0,5 \mathrm{~g} / \mathrm{L}$.

\section{Riegos}

Durante el periodo de siembra y de germinación se aplicó riego para mantener el sustrato húmedo a nivel de la semilla. La frecuencia fue de dos riegos diarios de 10 minutos en días despejados y de altas temperaturas, para mantener la humedad, temperatura y aireación óptimas para la germinación. En días nublados, se aplicó un riego diario de 5 minutos. Los riegos se realizaron en la mañana y por la tarde, evitando las altas temperaturas, con el objeto de reducir su evaporación.

Posterior a la germinación y hasta el mes de enero, los riegos se realizaran en forma diaria o día por medio por 10 minutos. Desde febrero hasta el mes de mayo los riegos fueron efectuados cada dos o tres días. El objeto de estas reducciones en los riegos es evitar la pérdida de nutrientes por exceso de agua y la aparición de musgos cercanos a la zona del cuello de la planta. Al igual que en el periodo de germinación, se mantiene el criterio de aplicación de riego por las mañanas.

\section{Fertilización}

Las fertilizaciones se realizaron con Dosatron, dosificador o inyector porcentual hidráulico que trabaja sin electricidad. El dosificador se instala directamente en la línea del suministro de agua, opera sin electricidad usando el volumen de agua que le entra como fuente de energía.

Al entrar el agua al dosificador activa el pistón, el cual a su vez activa la parte de la inyección que succiona el químico o concentrado de acuerdo al porcentaje o proporción escogido.

La cantidad de concentrado inyectado es directamente proporcional al volumen de agua que entra al dosificador, esta proporción se mantiene constante a pesar de las variaciones del caudal o de la presión que ocurran en la línea principal.

El tipo de fertilizante utilizado fue el de la línea Ultrasol de SOQUIMICH. Esta es una línea nutricional soluble en agua y libre de cloruro (excepto por Ultrasol TM MOP), lo que contribuye a una eficiente absorción de nutrientes. Las aplicaciones se realizaron con el siguiente criterio de acuerdo al estado de desarrollo de las plantas:

Hasta una altura promedio de $10 \mathrm{~cm}$ : Aplicación de Ultrasol Inicial (15-30-15) una vez por semana, en dosis de $2 \mathrm{~g} / \mathrm{L}$ de agua.

Entre los $10 \mathrm{~cm}$ y $25 \mathrm{~cm}$ de altura promedio: Aplicación de Ultrasol de Crecimiento (2510-10) alternando con Ultrasol de Desarrollo (18-6-18) una vez por semana, en dosis de $3 \mathrm{~g} / \mathrm{L}$. Se cambia el producto cada dos semanas. 
Sobre $25 \mathrm{~cm}$ de altura promedio: Aplicación de Ultrasol de Producción (13-6-40) en dosis de $2 \mathrm{~g} / \mathrm{L}$ de agua, una o dos veces por semana según estado de desarrollo.

También considera la aplicación de nitrato de calcio $2 \mathrm{~g} / \mathrm{L}$ de agua dos veces por semana y Coldkiller $2 \mathrm{cc} / \mathrm{L}$ de agua, una vez a la semana.

\section{Tratamientos Preventivos Contra Hongos}

Se utilizaron diversos fungicidas que se aplicaron con un intervalo de 15 días. Las aplicaciones se realizaron en las horas de bajas temperaturas, con bomba de espalda. La dosis utilizada fue de $1 \mathrm{~g} / \mathrm{L}$ litro de agua.

Captan, ingrediente activo Captan

Polyben, ingrediente activo Benomilo

Pomarsol, ingrediente activo Thiuram (TMTD)

Swift, ingrediente activo Triadimefon

Point Benomyl, ingrediente activo Benomilo

Dithane, ingrediente activo Mancozeb

\section{-Diseño Experimental}

Se consideró 10 tratamientos, dados por diversos tipos de contenedores, los cuales fueron representados cada uno en parcelas o bandejas compuestas por un número variable de plantas o repeticiones (Cuadro $\mathrm{N}^{\circ} 1$ ). Se utilizó 3 bloques.

\section{TRATAMIENTOS EVALUADOS EN PLANTAS DE QUILLAY}

\begin{tabular}{|c|c|c|c|c|c|c|}
\hline Tratamiento & Tipo Contendor & $\begin{array}{l}\text { Volumen } \\
\text { Cavidad } \\
\text { (cc) }\end{array}$ & $\begin{array}{l}\text { Cavidades } \\
\text { Almaciguera } \\
\left(\mathbf{N}^{\circ}\right)\end{array}$ & $\begin{array}{c}\text { Profundidad } \\
(\mathrm{mm})\end{array}$ & $\begin{array}{c}\text { Cavidades } \\
\left(\mathbf{N} / \mathbf{m}^{2}\right)\end{array}$ & Sección \\
\hline T1 & Almaciguera (13X8) & 56 & 104 & 70 & 416 & cuadrada \\
\hline $\mathrm{T} 2$ & Almaciguera $(15 \times 9)$ & 75 & 135 & 115 & 540 & cuadrada \\
\hline T3 & Almaciguera (14×8) & 95 & 112 & 140 & 448 & circular \\
\hline $\mathrm{T} 4$ & Almaciguera $(12 \times 7)$ & 100 & 84 & 100 & 336 & cuadrada \\
\hline T5 & Tubete $(14 \times 9)$ & 100 & 126 & 100 & 480 & cuadrada \\
\hline T6 & Almaciguera (12x7) & 130 & 84 & 160 & 336 & circular \\
\hline $\mathrm{T7}$ & Almaciguera (12x7) & 135 & 84 & 100 & 336 & cuadrada \\
\hline T8 & Tubete & 140 & 88 & 135 & 400 & circular \\
\hline T9 & Almaciguera $(10 \times 6)$ & 280 & 60 & 140 & 240 & cuadrada \\
\hline T10 & Almaciguera $(4 \times 6)$ & 310 & 24 & 150 & 233 & circular \\
\hline
\end{tabular}

\section{-Evaluación de Crecimiento}

En el mes de mayo de 2010, se realizó la medición de la altura total (Altura $\pm 0,1 \mathrm{~cm}$ ) y diámetro a la altura del cuello (DAC $\pm 0,01 \mathrm{~mm}$ ) de las plantas de quillay, así como la medición de la longitud de la raíz principal (Longitud raíces $\pm 0,1 \mathrm{~cm}$ ). Para la evaluación de estas variables, se 
consideraron distintos $\mathrm{n}$ (número de repeticiones, en función de las plantas centrales de cada bandeja)

Con el objeto de determinar parámetros de calidad de las plantas se estimaron algunos índices como el de Esbeltez (IE), que es el cociente entre la altura y el DAC (ALT/DAC).

Este índice relaciona la resistencia de la planta con la capacidad fotosintética de la misma (Toral 1997). Además, se utilizó relación diámetro : altura (DAC:ALT), esta relación está regulada en la Norma Chilena 2957 (INN 2006) para plantas distintas al quillay.

Otro Índice de calidad a evaluar es el de Dickson (QI) (Dickson et al. 1960), que integra la relación entre la masa seca total de la planta $(\mathrm{g})$ y la suma del cociente de esbeltez y la relación parte seca aérea/parte seca radical o Indice de Tallo-Raíz (ITR).

bloque.

Para la evaluación de estas variables se analizó 5 repeticiones de cada tratamiento y

Para analizar estadísticamente los datos, se utilizó el software estadístico INFOSTAT versión 20011/P (INFOSTAT, 2011).

A los datos obtenidos de cada variable, se les realizó un análisis de varianza tradicional. La homogeneidad de varianza fue evaluada mediante la prueba de Levene $(P<0,05)$. El supuesto de normalidad de los residuos se evaluó mediante la prueba de Shapiro-Wilks $(P<0,05)$. Para detectar diferencias significativas entre los tratamientos, se realizó la prueba de comparación múltiple Scott y Knott (1974), con un $95 \%$ de confianza, la cual permite comparar $\mathrm{n}$ distintos.

El modelo utilizado fue el siguiente:

$$
Y=\mu+B+T+\left(B^{*} T\right)+E
$$

Donde: $\quad Y=$ Altura $(\mathrm{cm})$; DAC $(\mathrm{mm})$; Longitud de raíz $(\mathrm{cm})$; Peso seco aéreo $(\mathrm{g})$;

Peso seco raíz (g); Peso seco total (g); Índice de Esbeltez (IE); Índice Tallo/Raíz (ITR); Índice de Calidad de Dickson (QI).

$\mu=$ Media

$\mathrm{B}=$ Bloque

$\mathrm{T}=$ Tratamiento (diferentes contenedores)

$\mathrm{E}=$ Error

\section{Crecimiento en Terreno}

\section{-Establecimiento}

La plantación se efectuó en el mes de Agosto del 2010 con plantas producidas en el vivero de INFOR en Concepción. El ensayo se encuentra ubicado en el sector San Lorenzo de la comuna de Florida, Provincia de Concepción, región del Bio Bio (36 47' 54"' LS; $72^{\circ} 39^{\prime}$ 61" LO, $238 \mathrm{msnm}$ ). Al ensayo se le realizó un cerco perimetral con alambre de púa, de tres hebras, para evitar el paso de animales mayores.

\section{-Espaciamiento y Preparación de Suelo}

Se realizó un roce liviano para despejar el sector del ensayo, en donde se encontraba vegetación arbustiva baja y desechos de cosecha de pinos, y el material de este roce fue ordenado en fajas. El trabajo de suelo consistió en un cultivo con azadón plantador removiendo el suelo a una profundidad de $40 \mathrm{~cm}$, con el fin de obtener un suelo mullido y suave, para posteriormente realizar la plantación en casillas de 40 × $40 \mathrm{~cm}$, espaciadas a 3 × 3m. 
La primera actividad de la plantación, consistió en cuadrar el terreno por medio de métodos prácticos de manera de lograr una perfecta alineación de la plantación. Una vez terminada la faena de plantación cada planta fue protegida con ramas para evitar el daño de conejo.

En el mes de septiembre de 2010 se realizó una fertilización con mezcla forestal, N 8\% P 20\% - K 7\%, con $140 \mathrm{~g}$ por planta depositada en 2 ranuras a una profundidad de $20 \mathrm{~cm}$ y a una distancia de $15 \mathrm{~cm}$ del cuello de la planta.

\section{-Diseño Experimental}

El diseño experimental correspondió a bloques completos al azar con tres repeticiones, considerando nueve volúmenes de contenedores (56, 75, 95, 100, 130, 135, 140, 280 y $310 \mathrm{~cm}^{3}$ ). La unidad muestral fue variable para cada volumen de contenedor. Se utilizó 10 tipos de contenedores (10 tratamientos), entre los cuales se probó 2 contenedores de igual volumen, 100 cc, uno de 84 cavidades y otro de 126 cavidades.

\section{-Evaluación de crecimiento}

Desde el establecimiento se ha realizado 2 evaluaciones, una en junio de 2011 (primera temporada de crecimiento) y otra en abril de 2012 (segunda temporada de crecimiento). Estas consistieron en una medición dasométrica, considerando las variables de altura total (Altura $\pm 0,1$ $\mathrm{cm}$ ) y diámetro a la altura del cuello (DAC $\pm 0,01 \mathrm{~mm}$ ), al total de plantas del ensayo.

Para analizar estadísticamente los datos, se utilizó el software estadístico INFOSTAT versión 20011/P (INFOSTAT, 2011).

A cada medición, se les realizó un análisis de varianza tradicional. La homogeneidad de varianza fue evaluada mediante la prueba de Levene $(P<0,05)$. El supuesto de normalidad de los residuos se evaluó mediante la prueba de Shapiro-Wilks $(P<0,05)$. Para detectar diferencias significativas entre los tratamientos, se realizó la prueba de comparación múltiple Scott y Knott (1974), con un $95 \%$ de confianza, la cual permite comparar $n$ distintos.

Se evalúo la correlación existente entre los parámetros morfológicos con el volumen del contenedor. Se identificó en cada caso las variables independientes significativamente correlacionadas con la variable de respuesta $\mathrm{P}<0,05$. Antes de realizar el análisis de la mortalidad, fue necesario transformar los valores de las variables medida expresada en porcentaje (\% de mortalidad) con el objetivo de que cumplieran con los supuestos de normalidad del modelo estadístico utilizado. Para esto se utilizó la transformación de Bliss, también conocida como transformación angular, que responde a la aplicación de la función: $Y^{\prime}=$ arc sen (raíz ( $\left.p / 100\right)$ ), donde $p$ es el valor en porcentaje de la variable observada (Ostle 1968; Box y Hunter, 1989).

El modelo utilizado fue el siguiente:

$$
Y=\mu+B+T+(B * T)+E
$$

Donde: $\quad \mathrm{Y}=$ Altura $(\mathrm{cm})$; DAC $(\mathrm{mm})$; Inc $\mathrm{H}$ incremento de altura $(\mathrm{cm})$; Inc Dac incremento de Dac (mm); Sobrevivencia (\%).

$\mu=$ Media

$\mathrm{B}=$ Bloque

$\mathrm{T}=$ Tratamiento (diferentes contenedores)

$\mathrm{E}=$ Error 


\section{RESULTADOS Y DISCUSIÓN}

\section{Crecimiento en Vivero}

Se puede apreciar en los Cuadros $\mathrm{N}^{\circ} 2$ y $\mathrm{N}^{\circ} 3$ que en general, los contenedores de mayor volumen $(280$ y $310 \mathrm{cc}$ ) presentaron valores medios de las variables superiores a los contenedores de menor volumen $(56,75$ y $95 \mathrm{cc})$.

Los resultados obtenidos en todos los tratamientos para la variable DAC y altura total de las plantas fueron superiores a los obtenidos por Viel (1999), quien analizando diversas procedencias de quillay de la región de Bio Bio obtuvo medias no superiores a 2,6 mm en DAC y $14,8 \mathrm{~cm}$ en altura, para plantas producidas en bolsas de aproximadamente $880 \mathrm{cc}$. Estos valores son similares a los registrados con el tratamiento T1, es decir, plantas producidas en el contenedor de menor volumen.

Para las variables de biomasa existe una directa relación con el volumen del contenedor (Cuadro $\mathrm{N}^{\circ} 3$ ). A mayor volumen de contenedor mayor peso seco aéreo y radicular. Solo el contenedor de $140 \mathrm{~cm}^{3}$ presenta un comportamiento diferente, presentando valores totales menores que los obtenidos en plantas producidas en contenedor de $135 \mathrm{~cm}^{3}$.

Respecto de esta característica algunos autores coinciden en la influencia que tiene la época de siembra sobre el peso total de la planta. Cabe señalar que la siembra fue realizada en junio de 2009 y que el peso total obtenido en todos los tratamientos fue superior a lo obtenido por Wiber (1991) donde obtuvo valores de 1,73 g para peso total de plantas de quillay sembradas en julio y valores inferiores de peso total para semillas sembradas en octubre $(0,8 \mathrm{~g})$; concordando con los estudios de Muñoz y Pérez (1981) en espino (Acacia caven (Mol.) Hooket) y algarrobo (Prosopis chilensis (Mol.) Stuntz), por lo que siembras más tempranas generan plantas de mayor biomasa.

\section{Cuadro $\mathrm{N}^{\circ} 2$}

EFECTO DEL VOLUMEN DEL CONTENEDOR SOBRE LOS ATRIBUTOS MORFOLÓGICOS EN PLANTAS DE QUILLAY PRODUCIDAS EN VIVERO $(n=15)$

\begin{tabular}{|c|c|cccc|ccccc|cccc|}
\hline Tratam. & $\begin{array}{c}\text { Volumen } \\
\text { Contenedor } \\
\text { (cc) }\end{array}$ & $\begin{array}{c}\text { Altura } \\
\text { (cm) }\end{array}$ & & DE & & $\begin{array}{c}\text { DAC } \\
\text { (mm) }\end{array}$ & & DE & & $\begin{array}{c}\text { Longitud } \\
\text { Raiz } \\
\text { (cm) }\end{array}$ & & DE & \\
\hline T1 & 56 & 14,86 & \pm & 2,54 & $\mathrm{c}$ & 2,85 & \pm & 0,34 & $\mathrm{~d}$ & 5,4 & \pm & 0,8 & $\mathrm{c}$ \\
T2 & 75 & 20,57 & \pm & 2,69 & $\mathrm{~b}$ & 3,62 & \pm & 0,8 & $\mathrm{c}$ & 9,55 & \pm & 2,01 & $\mathrm{~b}$ \\
T3 & 95 & 21,35 & \pm & 5,43 & $\mathrm{~b}$ & 4,08 & \pm & 0,67 & $\mathrm{~b}$ & 9,73 & \pm & 3,78 & $\mathrm{~b}$ \\
T4 & $100-84$ & 18,48 & \pm & 5,57 & $\mathrm{c}$ & 3,61 & \pm & 0,81 & $\mathrm{c}$ & 8,51 & \pm & 1,62 & $\mathrm{c}$ \\
T5 & $100-126$ & 22,75 & \pm & 5,54 & $\mathrm{~b}$ & 3,01 & \pm & 0,64 & $\mathrm{~d}$ & 7,52 & \pm & 2,51 & $\mathrm{c}$ \\
T6 & 130 & 20,83 & \pm & 6,48 & $\mathrm{~b}$ & 3,9 & \pm & 1,11 & $\mathrm{c}$ & 9,45 & \pm & 3,43 & $\mathrm{~b}$ \\
T7 & 135 & 28,57 & \pm & 5,23 & $\mathrm{a}$ & 4,37 & \pm & 0,96 & $\mathrm{~b}$ & 6,8 & \pm & 1,89 & $\mathrm{c}$ \\
T8 & 140 & 21,03 & \pm & 5,92 & $\mathrm{~b}$ & 3,55 & \pm & 0,95 & $\mathrm{c}$ & 9,18 & \pm & 4,6 & $\mathrm{~b}$ \\
T9 & 280 & 23,58 & \pm & 6,1 & $\mathrm{~b}$ & 4,82 & \pm & 0,68 & $\mathrm{a}$ & 11,86 & \pm & 2,25 & $\mathrm{a}$ \\
T10 & 310 & 29,37 & \pm & 8,31 & $\mathrm{a}$ & 4,97 & \pm & 0,64 & $\mathrm{a}$ & 12,7 & \pm & 1,89 & $\mathrm{a}$ \\
\hline
\end{tabular}

Medias con una letra común no son significativamente diferentes $(p<=0,05)$

DE: Desviación Estándar; DAC: Diámetro a la altura del Cuello 


\section{Cuadro $\mathrm{N}^{\circ} 3$ \\ EFECTO DEL VOLUMEN DEL CONTENEDOR SOBRE EL PESO SECO DE PLANTAS DE QUILLAY PRODUCIDAS EN VIVERO $(n=15)$}

\begin{tabular}{|c|c|cccc|cccc|cccc|}
\hline Tratam. & $\begin{array}{c}\text { Volumen } \\
\text { Contenedor } \\
\text { (cc) }\end{array}$ & $\begin{array}{c}\text { Peso Seco } \\
\text { Aéreo } \\
\mathbf{( g )}\end{array}$ & DE & & $\begin{array}{c}\text { Peso Seco } \\
\text { Raíz } \\
\mathbf{( g )}\end{array}$ & & DE & & \multicolumn{2}{|c|}{$\begin{array}{c}\text { Peso Seco } \\
\text { Total } \\
\text { (g) }\end{array}$} & DE & \\
\hline T1 & 56 & 1,51 & $\pm 0,41$ & $\mathrm{c}$ & 0,5 & \pm & 0,1 & $\mathrm{c}$ & 2,01 & \pm & 0,48 & $\mathrm{~d}$ \\
$\mathrm{~T} 2$ & 75 & 1,83 & $\pm 0,59$ & $\mathrm{c}$ & 0,76 & \pm & 0,23 & $\mathrm{c}$ & 2,58 & \pm & 0,77 & $\mathrm{~d}$ \\
$\mathrm{~T} 3$ & 95 & 1,92 & $\pm 0,85$ & $\mathrm{c}$ & 0,94 & \pm & 0,37 & $\mathrm{c}$ & 2,86 & \pm & 1,2 & $\mathrm{~d}$ \\
$\mathrm{~T} 4$ & $100-84$ & 1,94 & $\pm 0,84$ & $\mathrm{c}$ & 0,92 & \pm & 0,35 & $\mathrm{c}$ & 2,86 & \pm & 1,14 & $\mathrm{~d}$ \\
$\mathrm{~T} 5$ & $100-126$ & 2 & $\pm 0,75$ & $\mathrm{c}$ & 0,88 & \pm & 0,34 & $\mathrm{c}$ & 2,89 & \pm & 1,06 & $\mathrm{~d}$ \\
$\mathrm{~T} 6$ & 130 & 2,25 & $\pm 1,16$ & $\mathrm{c}$ & 1,35 & \pm & 0,76 & $\mathrm{~b}$ & 3,6 & \pm & 1,69 & $\mathrm{c}$ \\
$\mathrm{T} 7$ & 135 & 3,22 & $\pm 1,02$ & $\mathrm{~b}$ & 1,37 & \pm & 0,49 & $\mathrm{~b}$ & 4,59 & \pm & 1,37 & $\mathrm{~b}$ \\
$\mathrm{~T} 8$ & 140 & 2,25 & $\pm 0,99$ & $\mathrm{c}$ & 1,34 & \pm & 0,82 & $\mathrm{~b}$ & 3,59 & \pm & 1,49 & $\mathrm{c}$ \\
$\mathrm{T} 9$ & 280 & 3,53 & $\pm 1,15$ & $\mathrm{~b}$ & 1,75 & \pm & 0,48 & $\mathrm{~b}$ & 5,28 & \pm & 1,55 & $\mathrm{~b}$ \\
$\mathrm{~T} 10$ & 310 & 4,75 & $\pm 0,89$ & $\mathrm{a}$ & 2,23 & \pm & 0,58 & $\mathrm{a}$ & 6,98 & \pm & 1,34 & $\mathrm{a}$ \\
\hline
\end{tabular}

Medias con una letra común no son significativamente diferentes $(p<=0,05)$

DE: Desviación Estándar.

Respecto al índice de esbeltez, éste permite estimar la resistencia física de las plantas durante las operaciones de plantación y su resistencia al efecto mecánico del viento (Gómez y Leyva 2005).

Guifan (1997 cit. por García 2007) agrega que una planta de buena calidad debe tener un valor de diámetro de cuello relativamente alto y un índice de esbeltez bajo. En este estudio, el diámetro fue más bajo que el Índice de Esbeltez.

No obstante, Fernández y Royo (1998. cit. por García 2007) consideran que el valor de Esbeltez no debe ser mayor a 6 y Mitchel et al. (cit. por Hunt 1990) señalan que la esbeltez debe ser menor o igual a 8 para que la planta esté equilibrada.

Según ese criterio, todas las plantas de quillay evaluadas presentaron valores de esbeltez adecuados (Cuadro $\mathrm{N}^{\circ} 4$ ).

Respecto al QI, Hunt (1990), en estudios realizados en abeto y pino, señala que un valor inferior a 0,15 puede significar problemas en el establecimiento de las plantas, ya que estas se encontrarían desequilibradas, por ello para esas especies se recomienda un QI superior a 0,2 como mínimo.

Para esta especie, quillay, si bien es cierto no hay estudios que indiquen que QI es el ideal, todas las plantas de quillay obtuvieron un QI entre 0,24 y 0,88 (Cuadro $N^{\circ} 4$ ).

Al correlacionar las variables morfológicas, de biomasa y de calidad de las plantas con otras características de los contenedores, específicamente con su profundidad y densidad (№ de cavidades $/ \mathrm{m}^{2}$ ), se observa tendencias que resultan de interés, las cuales se resumen en el Cuadro $\mathrm{N}^{\circ} 5$.

Se puede observar en el Cuadro $N^{\circ} 5$ que variables como el DAC, biomasa e índice de calidad de Dickson, se correlacionan positiva y directamente con el volumen del contenedor. 
Respecto a las otras características del contenedor, se observa que solo el índice tallo/raíz se correlaciona positivamente y de forma directa con la profundidad del contenedor. En cuanto al número de cavidades por $\mathrm{m}^{2}$, las variables de biomasa e índice de calidad de Dickson se correlacionan positiva y directamente con esta variable.

\section{Cuadro $\mathrm{N}^{\circ} 4$}

EFECTO DEL VOLUMEN DEL CONTENEDOR SOBRE LOS INDICADORES DE CALIDAD DE PLANTAS DE QUILLAY PRODUCIDAS EN VIVERO $(n=15)$

\begin{tabular}{|c|c|cccc|ccccc|cccc|}
\hline Tratam. & $\begin{array}{c}\text { Volumen } \\
\text { Contenedor } \\
\text { (cc) }\end{array}$ & IE & & DE & & ITR & & DE & & INDICE QI & DE & \\
\hline T1 & 56 & 5,27 & \pm & 1 & $\mathrm{~b}$ & 3,06 & \pm & 0,72 & $\mathrm{a}$ & 0,24 & \pm & 0,05 & $\mathrm{c}$ \\
T2 & 75 & 5,94 & \pm & 1,46 & $\mathrm{~b}$ & 2,49 & \pm & 0,65 & $\mathrm{~b}$ & 0,33 & \pm & 0,14 & $\mathrm{c}$ \\
T3 & 95 & 5,19 & \pm & 0,78 & $\mathrm{~b}$ & 2,03 & \pm & 0,28 & $\mathrm{c}$ & 0,39 & \pm & 0,16 & $\mathrm{~b}$ \\
T4 & $100-84$ & 5,09 & \pm & 0,9 & $\mathrm{~b}$ & 2,17 & \pm & 0,69 & $\mathrm{~b}$ & 0,39 & \pm & 0,14 & $\mathrm{~b}$ \\
T5 & $100-126$ & 7,64 & \pm & 1,55 & $\mathrm{a}$ & 2,32 & \pm & 0,47 & $\mathrm{~b}$ & 0,3 & \pm & 0,13 & $\mathrm{c}$ \\
T6 & 130 & 5,51 & \pm & 1,6 & $\mathrm{~b}$ & 1,79 & \pm & 0,52 & $\mathrm{c}$ & 0,51 & \pm & 0,23 & $\mathrm{~b}$ \\
T7 & 135 & 6,69 & \pm & 1,19 & $\mathrm{a}$ & 2,43 & \pm & 0,58 & $\mathrm{~b}$ & 0,52 & \pm & 0,17 & $\mathrm{~b}$ \\
T8 & 140 & 6,02 & \pm & 1,32 & $\mathrm{~b}$ & 1,91 & \pm & 0,58 & $\mathrm{c}$ & 0,46 & \pm & 0,2 & $\mathrm{~b}$ \\
T9 & 280 & 4,9 & \pm & 1,13 & $\mathrm{~b}$ & 2,03 & \pm & 0,36 & $\mathrm{c}$ & 0,76 & \pm & 0,18 & $\mathrm{a}$ \\
T10 & 310 & 5,92 & \pm & 1,55 & $\mathrm{~b}$ & 2,23 & \pm & 0,54 & $\mathrm{~b}$ & 0,88 & \pm & 0,21 & $\mathrm{a}$ \\
\hline
\end{tabular}

Medias con una letra común no son significativamente diferentes $(p<=0,05)$

DE: Desviación Estándar. IE: Índice de Esbeltez ITR: Índice Tallo/Raíz

INDICE QI: Índice de Calidad de Dickson

Cuadro $\mathrm{N}^{\circ} 5$

CORRELACIÓN ENTRE LAS CARACTERÍSTICAS DEL CONTENEDOR SOBRE LOS ATRIBUTOS MORFOLÓGICOS, DE BIOMASA E INDICADORES DE CALIDAD DE PLANTAS DE QUILLAY PRODUCIDAS EN VIVERO

\begin{tabular}{|c|c|c|c|c|c|c|c|c|c|}
\hline \multirow{2}{*}{$\begin{array}{l}\text { Características } \\
\text { Contenedor }\end{array}$} & \multirow{2}{*}{$\begin{array}{l}\text { Altura } \\
\text { (cm) }\end{array}$} & \multirow{2}{*}{$\begin{array}{l}\text { DAC } \\
(\mathrm{mm})\end{array}$} & \multirow{2}{*}{$\begin{array}{c}\text { Longitud } \\
\text { Raíz } \\
\text { (mm) }\end{array}$} & \multicolumn{3}{|c|}{$\begin{array}{l}\text { Peso Seco } \\
\text { (g) }\end{array}$} & \multirow{2}{*}{ IE } & \multirow{2}{*}{ ITR } & \multirow{2}{*}{$\begin{array}{l}\text { INDICE } \\
\text { QI }\end{array}$} \\
\hline & & & & Aéreo & Raíz & Total & & & \\
\hline Volumen (cc) & 0,48 & 0,72 & 0,65 & 0,88 & 0,90 & 0,91 & 0,02 & 0,14 & 0,95 \\
\hline Profundidad $(\mathrm{mm})$ & 0,18 & 0,43 & 0,69 & 0,23 & 0,48 & 0,31 & 0,05 & 0,71 & 0,43 \\
\hline Cavidades $\left(\mathrm{N} / \mathrm{m}^{2}\right)$ & 0,23 & 0,54 & 0,25 & 0,62 & 0,66 & 0,65 & 0,12 & 0,10 & 0,73 \\
\hline
\end{tabular}

\section{Crecimiento en Terreno}

Los atributos morfológicos, según Rose et al. (1990), pueden correlacionarse exitosamente con la supervivencia y el crecimiento inicial de muchas especies forestales, por ello este estudio se evalúa los primeros años post plantación.

Se puede apreciar en el Cuadro $N^{\circ} 6$ que la mortalidad del ensayo en general es baja, se registra una mortalidad total inferior al $2 \%$ en el año 2011 y solo en el tratamiento T6 esta fue de un $6 \%$, En el año 2012 la mortalidad fue inferior al $5 \%$ en todo el ensayo, no obstante, el tratamiento T10 presentó una mortalidad de un $15 \%$. Este ensayo durante el año 2011 fue atacado 
por conejos, lo que generó que las plantas afectadas rebrotaran. Para efectos del análisis de la información, estas plantas dañadas no fueron consideradas. Estadísticamente no se observaron diferencias significativas entre los tratamientos al evaluar la supervivencia y el volumen del contenedor, no obstante, las plantas producidas en contenedores de 310, $280130 \mathrm{cc}$, presentaron los valores más bajos de supervivencia en términos porcentuales el año 2012.

\section{Cuadro $\mathrm{N}^{\circ} 6$}

EFECTO DEL VOLUMEN DEL CONTENEDOR EN LA MORTALIDAD DE LOS AÑOS 2011 Y 2012

\begin{tabular}{|c|c|c|c|c|c|c|c|c|c|c|}
\hline Trat & $\begin{array}{c}\text { Volumen } \\
\text { Contenedor } \\
\left(\mathrm{cm}^{3}\right)\end{array}$ & $\begin{array}{c}\mathbf{n} \\
\text { Original } \\
\left(\mathbf{N}^{\circ}\right)\end{array}$ & $\begin{array}{c}\text { Plantas } \\
\text { Eliminadas } \\
\text { análisis * } \\
\left(\mathrm{N}^{\circ}\right)\end{array}$ & $\begin{array}{c}\mathrm{n} \\
\text { Analizadas } \\
2011-2012 \\
\left(\mathrm{~N}^{\circ}\right)\end{array}$ & $\begin{array}{c}\text { Datos } \\
\text { Faltantes } \\
2011\end{array}$ & $\begin{array}{c}\text { Superv } \\
2011 \\
(\%)\end{array}$ & & $\begin{array}{c}\text { Datos } \\
\text { Faltantes } \\
2012\end{array}$ & $\begin{array}{c}\text { Superv. } \\
2012 \\
(\%) \\
\end{array}$ & \\
\hline 1 & 56 & 108 & 16 & 92 & 1 & 98,9 & a & 5 & $94,6 \%$ & $\mathrm{a}$ \\
\hline 2 & 75 & 108 & 13 & 95 & 1 & 98,9 & a & 5 & $94,7 \%$ & $\mathrm{a}$ \\
\hline 3 & 95 & 108 & 4 & 104 & 0 & 100,0 & a & 2 & $98,1 \%$ & $\mathrm{a}$ \\
\hline 4 & $100-84$ & 108 & 20 & 88 & 0 & 100,0 & a & 3 & $96,6 \%$ & $\mathrm{a}$ \\
\hline 5 & $100-126$ & 108 & 6 & 102 & 0 & 100,0 & a & 1 & $99,0 \%$ & $\mathrm{a}$ \\
\hline 6 & 130 & 108 & 9 & 99 & 6 & 93,9 & a & 7 & $92,9 \%$ & $\mathrm{a}$ \\
\hline 7 & 135 & 108 & 2 & 106 & 1 & 99,1 & a & 1 & $99,1 \%$ & $\mathrm{a}$ \\
\hline 8 & 140 & 108 & 12 & 96 & 3 & 96,9 & a & 6 & $93,8 \%$ & $\mathrm{a}$ \\
\hline 9 & 280 & 108 & 4 & 104 & 2 & 98,1 & a & 8 & $92,3 \%$ & $\mathrm{a}$ \\
\hline 10 & 310 & 48 & 1 & 47 & 1 & 97,9 & a & 7 & $85,1 \%$ & $\mathrm{a}$ \\
\hline
\end{tabular}

* Plantas eliminadas, corresponden a plantas cortadas o rebrotadas, no analizadas.

Medias con una letra común no son significativamente diferentes $(p<=0,05)$

Cuadro $\mathrm{N}^{\circ} 7$

EFECTO DEL VOLUMEN DEL CONTENEDOR EN EL DAC DE LOS AÑOS 2011 Y 2012 E INCREMENTO DEL DAC

\begin{tabular}{|c|c|c|c|c|c|c|c|c|c|c|c|c|c|c|c|c|}
\hline Trat. & $\begin{array}{c}\text { Volumen } \\
\text { Contenedor } \\
\left(\mathrm{cm}^{3}\right)\end{array}$ & $\mathbf{n}$ & $\begin{array}{l}\text { DAC } \\
2011 \\
(\mathrm{~mm}) \\
\end{array}$ & & DE & & $\mathbf{n}$ & $\begin{array}{l}\text { DAC } \\
2012 \\
(\mathrm{~mm}) \\
\end{array}$ & & DE & & $\mathbf{n}$ & $\begin{array}{c}\text { Inc } \\
\text { DAC } \\
(\mathrm{mm})\end{array}$ & & DE & \\
\hline 1 & 56 & 91 & 5,07 & \pm & 1,72 & c & 87 & 6,5 & \pm & 3,35 & $d$ & 65 & 2,18 & \pm & 1,93 & $b$ \\
\hline 2 & 75 & 94 & 5,22 & \pm & 1,12 & c & 90 & 6,04 & \pm & 1,85 & $d$ & 55 & 1,65 & \pm & 1,06 & $b$ \\
\hline 3 & 95 & 104 & 5,46 & \pm & 1,05 & c & 102 & 6,95 & \pm & 2,06 & C & 76 & 2,13 & \pm & 1,59 & $b$ \\
\hline 4 & $100-84$ & 88 & 5,74 & \pm & 1,47 & b & 85 & 7,39 & \pm & 2,59 & $\mathrm{C}$ & 61 & 2,5 & \pm & 1,61 & $a$ \\
\hline 5 & $100-126$ & 102 & 5,8 & \pm & 1,27 & c & 101 & 7,26 & \pm & 2,12 & C & 68 & 2,28 & \pm & 1,37 & $\mathrm{~b}$ \\
\hline 6 & 130 & 93 & 5,94 & \pm & 1,7 & $b$ & 92 & 8,05 & \pm & 3 & D & 75 & 2,74 & \pm & 2,03 & a \\
\hline 7 & 135 & 105 & 6,19 & \pm & 1,52 & $b$ & 105 & 8,05 & \pm & 2,34 & $b$ & 88 & 2,39 & \pm & 1,68 & a \\
\hline 8 & 140 & 93 & 5,32 & \pm & 1,62 & C & 90 & 6,5 & \pm & 2,48 & d & 65 & 1,96 & \pm & 1,47 & $b$ \\
\hline 9 & 280 & 102 & 5,89 & \pm & 1,25 & $b$ & 96 & 7,28 & \pm & 2,3 & C & 70 & 1,97 & \pm & 1,56 & $\mathrm{~b}$ \\
\hline 10 & 310 & 46 & 7,02 & \pm & 1,84 & a & 40 & 9,13 & \pm & 3,07 & $a$ & 31 & 3,1 & \pm & 2,02 & $\mathrm{a}$ \\
\hline
\end{tabular}

Medias con una letra común no son significativamente diferentes $(p<=0,05)$

DE: Desviación Estándar.

Inc. DAC: Incremento en DAC 
En cuanto al comportamiento del DAC, se puede observar en el Cuadro $\mathrm{N}^{\circ} 7$ que para el año 2011, como para el año 2012, los valores más altos fueron obtenidos por plantas producidas en contenedores de $310 \mathrm{cc}$, seguidos por plantas producidas en contendores de 135 y $130 \mathrm{cc}$, siendo estos valores estadísticamente diferentes respecto a los otros tratamientos (Figura $\mathrm{N}^{\circ} 1$ ).

Las plantas producidas en contenedores de 56 y 75 cc presentaron los valores más bajos de DAC, tanto en el año 2011 como en el 2012.

La misma tendencia se observa respecto al incremento en altura. Considerando que estas plantas fueron establecidas en una zona de secano interior y que no han sido regadas, se observa un crecimiento sostenido desde que fueron establecidas, alcanzando el año 2011 medias de DAC que oscilaron entre 5,07 y 7,02 mm; el DAC medio del año 2012 osciló entre los 6,04 y $9,13 \mathrm{~mm}$.

En el Cuadro $N^{\circ} 8$ se presenta los valores de altura obtenidos en el año 2011 y 2012 . Se aprecia que al igual que en el caso del DAC, esta variable manifiesta la misma tendencia, es decir a medida que aumenta el volumen del contenedor la altura de la planta aumenta, excepto en los contenedores de 140 y $280 \mathrm{cc}$ (Figura $\mathrm{N}^{\circ} 1$ ).

Respecto del incremento en altura, se observa incrementos importantes en los tratamientos T8 (280 cc), T1 (56 cc) y T4 (100 cc y 84 cavidades), lo que supone una tendencia a la homogeneización de las alturas medias, entre los tratamientos a medida que pasan los años.

Estos resultados son coincidente con lo obtenido Lamhamedi et al. (1998), quienes demostraron que un contenedor con mayor capacidad volumétrica favorece el desarrollo de las plantas.

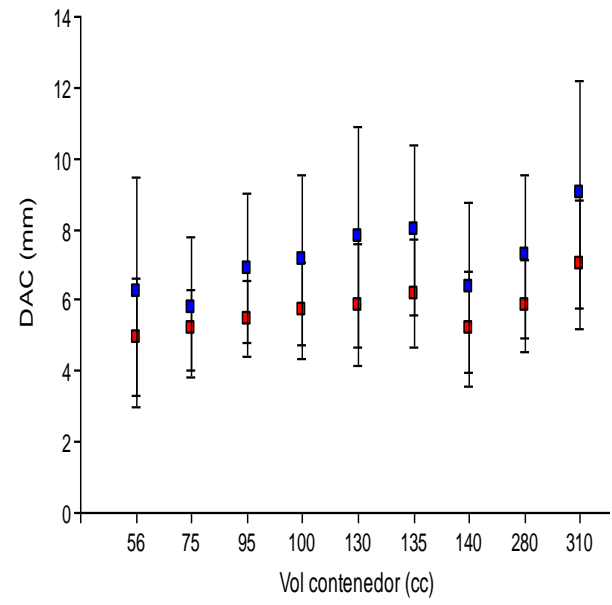

DAC2011 $\square$ DAC2012

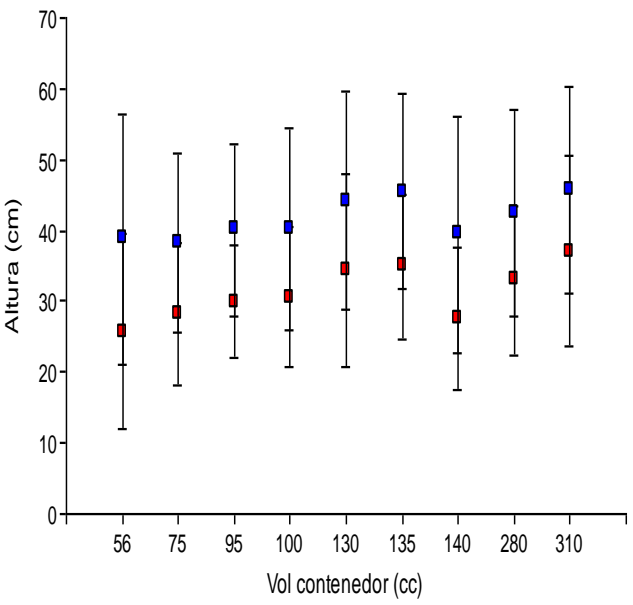

Altura $2011 \square$ Altura 2012

Figura $N^{\circ} 1$

COMPORTAMIENTO DE LA ALTURA Y DAC DE PLANTAS DE QUILLAY

EN LOS AÑOS 2011 Y 2012 


\section{Cuadro $\mathrm{N}^{\circ} 8$ \\ EFECTO DEL VOLUMEN DEL CONTENEDOR EN LA ALTURA DE LOS AÑOS 2011 Y 2012 E INCREMENTO DE LA ALTURA}

\begin{tabular}{|c|c|c|c|c|c|c|c|c|c|c|c|c|c|c|c|c|}
\hline Trat. & \begin{tabular}{|c|}
$\begin{array}{c}\text { Volumen } \\
\text { Contenedor } \\
\left(\mathrm{cm}^{3}\right)\end{array}$ \\
\end{tabular} & $\mathbf{n}$ & $\begin{array}{l}\text { Altura } \\
2011 \\
\text { (cm) }\end{array}$ & & DE & & $n$ & $\begin{array}{l}\text { Altura } \\
2012 \\
\text { (cm) }\end{array}$ & & DE & & $n$ & $\begin{array}{c}\text { Inc } \\
\text { ALT } \\
(\mathrm{cm}) \\
\end{array}$ & & DE & \\
\hline 1 & 56 & 91 & 26,85 & \pm & 14,26 & c & 87 & 41,73 & \pm & 16,72 & 0 & 86 & 14,62 & \pm & 10,00 & a \\
\hline 2 & 75 & 94 & 28,49 & \pm & 10,17 & c & 90 & 39,45 & \pm & 11,98 & $b$ & 81 & 12,32 & \pm & 9,87 & $\mathrm{~b}$ \\
\hline 3 & 95 & 104 & 30,28 & \pm & 7,68 & c & 102 & 40,69 & \pm & 11,84 & b & 98 & 11.00 & \pm & 8,54 & $\mathrm{~b}$ \\
\hline 4 & $100-84$ & 88 & 30,07 & \pm & 10,36 & c & 85 & 42,16 & \pm & 14,75 & $\mathrm{~b}$ & 78 & 13,22 & \pm & 11,88 & a \\
\hline 5 & $100-126$ & 102 & 32,14 & \pm & 9,06 & $b$ & 101 & 40,62 & \pm & 12,92 & $b$ & 89 & 10,42 & \pm & 11,52 & $b$ \\
\hline 6 & 130 & 93 & 35,05 & \pm & 13,49 & $a$ & 92 & 45,75 & \pm & 14,59 & a & 86 & 12,23 & \pm & 12,16 & $b$ \\
\hline 7 & 135 & 105 & 35,14 & \pm & 9,85 & $a$ & 105 & 45,76 & \pm & 13,58 & a & 101 & 11,18 & \pm & 7,94 & $\mathrm{~b}$ \\
\hline 8 & 140 & 93 & 28,16 & \pm & 9,75 & c & 90 & 42,34 & \pm & 15,31 & b & 84 & 15,95 & \pm & 13,06 & $\mathrm{a}$ \\
\hline 9 & 280 & 102 & 33,36 & \pm & 10,1 & $b$ & 96 & 43,05 & \pm & 14,18 & b & 91 & 9,94 & \pm & 9,50 & $b$ \\
\hline 10 & 310 & 46 & 37,46 & \pm & 13,35 & $\mathrm{a}$ & 40 & 46,41 & \pm & 13,95 & $\mathrm{a}$ & 33 & 11,74 & \pm & 9,59 & $\mathrm{~b}$ \\
\hline
\end{tabular}

Medias con una letra común no son significativamente diferentes $(p<=0,05)$

DE: Desviación Estándar.

Inc. ALT: Incremento en Altura

Como se puede observar en el Cuadro $\mathrm{N}^{\circ} 9$, a pesar que los valores de correlación no son tan altos como los obtenido por González et al. (2011), en plantas de quillay de una temporada producidas en vivero, se puede señalar que la altura y DAC de las plantas de quillay luego de dos años de establecidas aumentan a medida que aumenta el volumen del contenedor. No obstante, el valor de esta correlación disminuye, a medida que transcurre el tiempo desde que las plantas fueron retiradas del vivero.

Esto coincide con lo manifestado por Pinto et al. (2011), que evaluaron el comportamiento de Pinus ponderosa en dos sitios, uno mediterráneo y otro más árido, concluyendo que las características iniciales de las plántulas pierden su valor predictivo con el tiempo, sobre todo en el sitio de tipo Mediterráneo, en donde el crecimiento absoluto es mayor que en el otro sitio, pero el contenedor induce características, las que se ven limitadas por factores ambientales y genéticos. A la inversa, en un sitio más árido, donde el crecimiento absoluto se redujo, los rasgos determinados por el tipo de contenedor persistieron por más tiempo.

Lo obtenido concuerda con lo señalado por Domínguez et al. (1997) y Domínguez et al. (2000), quienes indican que el tamaño del contenedor tiene una correlación directa con los parámetros morfológicos de las plantas que se producirán. A mayor volumen del contenedor, las magnitudes de altura y diámetro de cuello también aumentan.

\section{Cuadro $\mathrm{N}^{\circ} 9$}

\section{COEFICIENTE DE CORRELACIÓN DE PEARSON ENTRE LOS PARÁMETROS MORFOLÓGICOS} Y SOBREVIVENCIA Y EL VOLUMEN DEL CONTENEDOR

\begin{tabular}{|c|c|c|c|c|c|c|c|}
\hline \multicolumn{2}{|c|}{ DAC } & \multicolumn{2}{c|}{ Altura } & \multirow{2}{*}{$\begin{array}{l}\text { Incremento } \\
\text { DAC }\end{array}$} & $\begin{array}{c}\text { Incremento } \\
\text { Altura }\end{array}$ & \multicolumn{2}{c|}{ Supervivencia } \\
& 2011 & 2012 & 2011 & 2012 & & 2011 & 2012 \\
\hline 0,78 & 0,69 & 0,72 & 0,69 & 0,47 & $-0,41$ & $-0,23$ & $-0,77$ \\
\hline
\end{tabular}


Al respecto, Close et al. (2010) señalan, en un estudio realizado con plantas de Eucalyptus globulus, que cuando estas son producidas en contenedores de menor volumen, con sistemas radiculares poco desarrollados, conducen a un menor crecimiento, sin embargo, contenedores con mayor volumen y profundidad mejoran el crecimiento de las plantas en terreno, aumentando la absorción de humedad desde el suelo a través de las raíces.

Esto también ha sido observado en plantas de Pinus pinea L. por Rincón (1998), quien señala que plantas producidas en contenedores de $300 \mathrm{cc}$ crecen mejor en terreno que plantas producidas en contenedores de $175 \mathrm{cc}$, las cuales disminuyen el crecimiento de pino piñonero en campo. En esta misma especie, Cañellas et al. (1999) señalan que en ensayos de técnicas de cultivo de plantas para evaluar tipo y tamaño de contenedor, dosis de fertilizante y densidad de producción de planta, no se encontró efectos sobre la supervivencia de las plantas, pero si en el crecimiento de las mismas. Se ha obtenido mayor crecimiento de las plantas (longitud del tallo, raíz y peso seco aéreo y radical) en los contenedores de mayores dimensiones y con mayor concentración de fertilizante. Lla selección del contenedor más apropiado dependerá del balance entre la calidad de la planta producida y el costo de su producción (Arizaleta y Pire 2008).

Con los resultados obtenidos a la fecha se puede concluir que plantas producidas en contenedores de 130 y $135 \mathrm{cc}$, presentan parámetros morfológicos, al cabo de dos años de establecidas las plantas en terreno, superiores a los de contenedores de mayor y menor volumen, excepto volúmenes de $310 \mathrm{cc}$. Cabe señalar que producir plantas en este último tipo de contenedor es más costoso que producir plantas en aquellos de 130 y $135 \mathrm{cc}$, que presentan parámetros morfológicos igualmente interesantes a costos más bajos. Estos resultados difieren de otros obtenidos con especies de crecimiento más rápido como Eucalyptus o Acacia, en las cuales el efecto del tamaño de contenedor utilizado pierde significancia en terreno (INFOR-CTPF, 2012 datos no publicados y en prensa, respectivamente). No obstante, en especies de crecimiento más lento como es quillay, luego de dos años de establecidas las plantas en terreno es posible encontrar diferencias de crecimiento atribuibles al tipo de contenedor utilizado en la producción de plantas.

Se observa además en el Cuadro $N^{\circ}$ 9, la correlación negativa que existe entre la supervivencia del año 2012 y el volumen del contenedor, lo que indicaría que a medida que el volumen del contenedor es mayor, disminuye la supervivencia. Esta correlación no se observó en el año 2011. Es necesario cotejar los valores observados en el Cuadro $\mathrm{N}^{\circ} 9$ con evaluaciones posteriores para poder determinar tendencias para cada una de las variables evaluadas.

\section{CONCLUSIONES}

El volumen del contenedor es la característica de este que se correlaciona mejor con las variables morfológicas de las plantas producidas en vivero, siendo el peso seco y el DAC las variables con una mayor correlación y esta se da principalmente dentro de un rango bien definido de volumen.

Existe una alta correlación con las variables morfológicas de las plantas en etapa de vivero, es decir a medida que aumenta el volumen y profundidad del contenedor, mayores son los valores de dichas variables, característica que se presenta con mayor notoriedad en la longitud de la raíz. Por el contrario, estas variables disminuyen a medida que aumenta el número de cavidades por almaciguera y por unidad de superficie, principalmente en la altura y el diámetro.

Luego de dos años de establecido el ensayo de quillay en la comuna de Florida en la región del Biobío, se concluye que durante el último periodo se observa una relación entre el tamaño de la planta de Quillaja saponaria (dada por el volumen del contenedor) establecida en terreno y su supervivencia, la cual al segundo año es superior al $95 \%$. Los volúmenes de los contenedores afectaron significativamente el crecimiento en diámetro y la altura de las plantas, tanto durante el primer como el segundo año de establecido el ensayo. En general, ambas 
variables aumentan a medida que se incrementan los volúmenes de los contenedores, excepto en los contenedores de 140 y $280 \mathrm{cc}$, los que presentan valores inferiores a los contenedores de 135 cc. Las correlaciones entre el volumen del contenedor y los parámetros morfológicos de las plantas de quillay disminuyen, a medida que transcurre el tiempo desde que las plantas fueron retiradas del vivero.

A la luz de los resultados obtenidos, luego de dos años de establecidas las plantas de quillay en terreno, pareciera ser más rentable y productivo producir plantas de quillay en contenedores de volumen entre 130 y $135 \mathrm{cc}$. Evaluaciones posteriores permitirán apreciar si las diferencias se mantienen a mayor edad de la plantación.

\section{REFERENCIAS}

Arizaleta, M. y Pire, R., 2008. Respuesta de plántulas de cafeto al tamaño de la bolsa y fertilización con nitrógeno y fósforo en vivero. Agrociencia 42: 47-55.

Box, G. y Hunter, W., 1989. Estadística para investigadores. Introducción al diseño de experimentos, análisis de los datos y construcción de modelos. U.S.A. Ed. Reverté S.A. 675 p.

Cañellas, I., Finat, L., Bachiller, A. y Montero, G., 1999. Comportamiento de plantas de Pinus pinea en vivero y campo: Ensayos de técnicas de cultivo de plantas, fertilización y aplicación de herbicidas. Invest. Agr.: Prod. Prot. Veg. Vol. 8 (2): 335-359.

Close, D., Paterson, S., Corkrey, R. y Mc Arthur, C., 2010. Influence of seedling size, container type and mammal browsing on the establishment of Eucalyptus globulus in plantation forestry. New Forests 39: 105-115.

Dickson, A., Leaf, Al., Hosner, I., 1960. Quality appraisal of White Spruce and White Pine seedlings stock in nurseries. Forest Chronicle 36: 10-13.

Domínguez, S., 1997. La importancia del envase en la producción de plantas forestales. Quercus 134: 34-37.

Domínguez, S., 2000. Influencia de distintos tipos de contenedores en el desarrollo en campo de Pinus halepensis y Quercus ilex. Reunión de Coordinación I+D. Fundación CEAM.

Domínguez, S., Herrero, N., Carrasco, I., Ocaña, L. y Peñuelas, J., 1997. Ensayo de diferentes tipos de contenedores para Quercus ilex, Pinus halepensis, Pinus pinaster y Pinus pinea: Resultados de vivero. Actas del II Congreso Forestal Español. 1997. Pamplona. Mesa 3: 189-194

Domínguez, S., Carrasco, I., Herrero, N., Ocaña, L., Nicolás, L.y Peñuelas, J., 2000. Las características de los contenedores influyen en la supervivencia y crecimiento de las plantas de Pinus pinea en campo. Actas del 1er Simposio sobre el pino piñonero. 2000. Valladolid. Volumen 1: 203-209.

Donoso, S., Peña, K., Pacheco, C., Luna, G. y Aguirre, A., 2011. Respuesta fisiológica y de crecimiento en plantas de Quillaja saponaria y Cryptocarya alba sometidas a restricción hídrica. Bosque 32(2): 187-195.

Enciclopedia de la Flora Chilena, 2012. Quillaja saponaria Mol. Quillay. Disponible en: http://museo.florachilena.cl/Niv_tax/Angiospermas/Ordenes/Fabales/Quillajaceae/Quillay.htm

García, M. A., 2007. Importancia de la calidad del plantín forestal. XXII Jornadas Forestales de Entre Ríos. Argentina, Octubre de 2007. Disponible en:

http://www.inta.gov.ar/concordia/info/Forestales/contenido/pdf/2007/312.II.GARCIA.pdf (con acceso el 15-6-2010).

Gómez, Y. y Leyva, I., 2005. Producción de plantas de Eucalyptus grandis en viveros, mediante la obtención de un sustrato utilizando como elemento principal la cachaza. Revista Electrónica Hombre Ciencia y Tecnología, Guantánamo, Cuba. Volumen 33: 10p. Disponible en:

http://www.gtmo.inf.cu/revista\%20electronica/numero_33/Producci\%F3n\%20de\%20planta.pdf (con acceso el 23 de julio de 2008).

González, M., Quiroz, I., García, E. y Soto, H., 2011. Estándares de producción de plantas de Quillay (Quillaja saponaria Mol.) Revista Chile Forestal 353: $43-46$. 
Hunt, G. A., 1990. Effect of styroblock design and copper on morphology of conifer seedlings. En: Rose, R., S. J. Campbell y T. D. Landis (eds.). Proceedings, Western Forest Nursery Association; 1990 August 13-17; Roseburg, OR. General Technical Report RM-200. Fort Collins, CO: U.S. Department of Agriculture, Forest Service, Rocky Mountain Forest and Range Experiment Station: 218-222. Disponible www.rngr.net/publications/proceedings/1990/hunt.pdf/at_download/file (con acceso el 30 de julio de 2008).

INFOR-INDAP-FIA, 2000. Monografía Quillay (Quillaja saponaria). Diversificación de Alternativas de Producción Forestal y Agroforestal para Pequeños Propietarios en el Secano. Proyecto de Desarrollo de las Comunas Pobres de la Zona del Secano (PRODECOP-Secano).

INN, 2006. Norma Chilena Oficial. NCh 2957. Of2006. Material de propagación de uso forestal. Instituto Nacional de Normalización 1ra ed. Santiago, Chile.

INFOSTAT, 2011. InfoStat versión 2011. Grupo InfoStat, Facultad de Ciencias Agrarias, Universidad Nacional de Córdoba. Córdoba, Argentina.

Lamhamedi, M., Bernier, P., Hebert, C. and Jobidon, R., 1998. Physiological and growth responses of three sizes of containerized of Picea mariana seedlings outplanted with and without vegetation control. Forest Ecology and Management 110: 13-23.

Muñoz, A. y Perez, A., 1981. Factores que influyen en la producción de plantas de Acacia caven (Mol.) Hooket y Prosopis chilensis (Mol.) Stuntz. Tesis de Grado. Universidad de Chile. Facultad de Ciencias Agrarias y Forestales.137 p.

Ostle, B., 1968. Estadística Aplicada. Técnicas de la Estadística Moderna, cuando y donde aplicarlas. México. Editorial Limusa - Wiley, S. A. 629 p.

Pinto, J., Marshall, J., Dumroese, K., Davis, A. and Cobos, D. 2011. Establishment and growth of container seedlings for reforestation: A function of stocktype and edaphic conditions. Forest Ecology and Management. Volume 261, Issue 11, 1 June 2011, Pages 1876-1884.

Rincón, A. M., 1998. Identificación y evaluación de hongos para la micorrización controlada de Pinus pinea L. producido en contenedor. Tesis Doctoral. Facultat de Ciencies, Universitat Autònoma de Barcelona, Barcelona.

Rodríguez , R, Matthei, S. y Quezada, M., 1983. Flora arbórea de Chile. Concepción, Chile. Editorial de la Universidad de Concepción. 408 p.

Rose, R., Carlson, W. y Morgan, P., 1990. The target seedling concept. Chapter 1. In: Rose R, S Campbell, T Landis (eds.) Target Seedling Symposium. Roseburg, Oregón. 275 p.

Santelices, R. y Bobadilla, C., 1997. Arraigamiento de estacas de Quillaja saponaria Mol. Y Peumus boldus Mol. Bosque 18 (2): $77-85$.

Scott, A. J. and Knott, M., 1974. A cluster analysis method for grouping means in the analysis of variance. Biometrics, 30: 507-512

Toral, I., 1997. Concepto de calidad de plantas en viveros forestales. Documento Técnico 1. Programa de Desarrollo Forestal Integral de Jalisco. Guadalajara, México. 26 p.

Viel, D., 1999. Caracterización de plantas de Quillaja saponaria Mol. provenientes de semillas de distintas procedencias de la Octava Región. Memoria para optar al título profesional de Ingeniero Forestal. Universidad de Concepción. Facultad de Ciencias Forestales. 40 p.

Wiber, K. S., 1991. Factores que influyen en la germinación y producción de plantas de Quillay (Quillaja saponaria Mol.). Tesis Ing. Forestal. Santiago, Universidad de Chile, Fac. de Cs Agr. y For. 133p. 
